Clement was one of the first to address many questions that have engaged Christian thinkers through the centuries - what is the relation of faith and reason, of divine revelation and human thinking, of Scripture and philosophy? What is the purpose of human life; who is God and how is he known; how is one's faith to be lived out in practice? He began a particular tradition of exegetical and theological reflection that is evident especially in the Greek-speaking East, for example in Origen, Gregory of Nyssa, Gregory of Nazianzus, Basil, Evagrius, and Maximus the Confessor, and also in Latin Fathers such as Ambrose. Rescue from sin is a necessary preliminary stage in salvation, but at the center of Clement's soteriology and eschatology is the knowledge and love of God. His most significant legacy for later patristic theology is his insistence on life-long seeking for greater understanding — in short, his call for Christian theologizing. One of his favorite biblical verses is Matt 7:7: Seek and you shall find. Clement is confident that all paths in the human search for truth can contribute to this final goal - and that the best achievements of human wisdom are in fact part of the divine oikovouia, God's comprehensive plan for bringing all human beings to himself.

\title{
Basilius von Cäsarea (329?-378?) in der Polyphonie der Kirchenväter
}

\author{
Johannes Zachhuber
}

\begin{abstract}
The article offers a brief presentation of Basil of Caesarea (329-378) in the context of a polyphony of the church fathers. Basil, one of the most influential figures in the entire history of the Eastern Church, held close contacts with many other major theologians and bishops of his time. His large corpus of extant letters gives a strong sense of his participation in a polyphonic interaction between diverse Christian voices. Yet he is also elusive and often difficult to integrate into conventional categories of patristic history. This can be explained by his pragmatic approach to theology which uniquely combines deep intellectual sophistication with a level-headed awareness of the practical needs of the Church in his own time.
\end{abstract}

\section{Vorbemerkung}

Die Metapher der >Polyphonie kann auf mindestens zweierlei Weise verstanden werden. Einerseits verweist sie auf die Vielzahl selbstständiger, gleichwohl miteinander verflochtener und verwobener Stimmen, die in irreduzibler Pluralität das

Evang. Theol. 79. Jg., Heft 5, S. 359-369, ISSN 0014-3502

(C) 2019 by Gütersloher Verlagshaus, Gütersloh, in der Verlagsgruppe Random House GmbH, München 
spätantike Christentum ausmachen. Das Christentum jener Zeit bestand nicht aus isolierten Einzelfiguren, zu denen eine immer stärker spezialisierte Patristik die von ihr untersuchten Autoren manchmal macht. Ebenso wenig jedoch war die christliche Kirche der Spätantike eine undifferenzierte Einheit, wie es das ekklesiologische Konstrukt der >Kirchenväter`suggeriert. Vielmehr müssen alle am damaligen theologischen Diskurs Beteiligten als eigenständige Denker und Akteure mit je besonderen Fragestellungen, Ideen und Interessen gewürdigt, gleichzeitig aber auch im Zusammenhang mit dem Handeln und Denken anderer Personen in ihrem geographischen und historischen Umfeld verstanden werden. Diese fundamentale aber oft ignorierte Tatsache wird durch den Begriff der Polyphonie treffend eingefangen und auf den Begriff gebracht.

Das Wort von der Polyphonie kann freilich auch andere Assoziationen auslösen; dann nämlich, wenn man primär an die Einheit und Einheitlichkeit des polyphonen Musikstückes denkt. Spricht man von der patristischen Theologie als einer Polyphonie in diesem Sinn, dann fragt man sogleich - um im Bild zu bleiben - wer der Komponist des Stückes ist, wer die Partitur schrieb, nach der die einzelnen zu spielen hatten, und wer das Orchester oder den Chor mit seinen Einzelstimmen dirigierte. Anders gesagt, statt Pluralität und Vielfalt der christlichen Stimmen in der Spätantike herauszustellen, kann die Metapher von der Polyphonie diese Pluralität auch relativieren und geradezu in den Hintergrund treten lassen. Dann ergibt sich die Gefahr, dass die Individualität der einzelnen Theologen aus dem Blick gerät und die Geschichte als überindividueller Prozess erscheint, in dem den Einzelnen nur eine untergeordnete, funktionale Bedeutung zukam, wie es etwa in der Fluchtlinie des hegelschen oder des marxistischen Geschichtsverständnisses liegt.

Ich kann die Problematik, die sich aus diesem doppelten Verständnis der Leitmetapher ergibt, hier nicht ausführlich diskutieren und begnüge mich daher mit der Feststellung, dass ich im Folgenden Polyphonie in der ersten der beiden Bedeutungen verstehen werde. In diesem Sinn geht es mit darum, die Person des Basilius von Cäsarea dadurch besser in den Blick zu bekommen, dass ich seine eigene Arbeit mit der anderer Denker der patristischen Epoche in Verbindung setze. Dabei bin ich mir bewusst, dass ein solcher Versuch schnell zur Postulierung problematischer Kategorien führen kann; die Forschungsgeschichte wimmelt geradezu von solchen: Gnostiker, Origenisten, Alexandriner, Antiochener - sie alle werden von den Forschern einer Generation eingeführt nur um von ihren Nachfolgern als einseitig oder irreführend kritisiert und mit Aplomb verabschiedet zu werden. Vor solchen Generalisierungen also gilt es auf der Hut zu sein; wie wir sehen werden, tragen sie im Fall des Basilius ohnehin wenig aus.

\section{Ein Zugang zu Basilius in der Polyphonie der Kirchenväter}

Wie leicht oder schwer ist es, das Konzept der Polyphonie in dem gerade skizzierten Sinn auf Basilius anzuwenden? Die etwas paradoxale Antwort lautet, dass es sowohl ungewöhnlich leicht als auch ungewöhnlich schwer ist. Leicht ist es, sofern es sich bei Basilius um eine der am besten vernetzten Personen der patristischen Zeit überhaupt handelt. Zumindest, so muss man wohl angesichts der schwierigen Quellenlage einschränkend hinzufügen, ist er einer derjenigen, über dessen Vernetzung wir besonders gut unterrichtet sind. Das verdanken wir vor allem dem großen Schatz 
seiner Korrespondenz, die uns in weit über 300 Briefen erhalten ist. ${ }^{1}$ Jeder Versuch, sich der Person des Basilius zu nähern - und darüber hinaus auch fast alle Versuche, die Kirchengeschichte seiner Zeit zu schreiben - beginnen und enden mit der genauen Analyse der Informationen, die wir über ihn selbst und seine diversen Freundschaften, Kollaborationen, Konflikte und Rivalitäten aus dieser Fundgrube gewinnen können. Es ist kein Zufall, dass ein wichtiges Buch zu Basilius in seinem Titel vom »Kosmos seiner Freunde « spricht, der aus seiner Korrespondenz zu erheben ist. ${ }^{2}$

Aus diesen Briefen bekommt man einen überaus plastischen Eindruck von dem vielfältigen Beziehungsgeflecht, in das der Heilige eingebunden war - besonders gilt das für seine letzten Lebensjahre, von der Ernennung zum Metropoliten von Cäsarea 370 bis zu seinem vorzeitigen Tod, der wohl in das Jahr 378 zu datieren ist. ${ }^{3}$ In erster Linie erhellen die erhaltenen Briefe seine engen allerdings auch hochkomplizierten Beziehungen zu seinem Studienfreund Gregor von Nazianz (329-390) und zu seinem jüngeren Bruder, Gregory von Nyssa - für die byzantinische Nachwelt waren diese bald die >drei großen Kappadozier', ein Beiname, den trotz wiederholter Kritik auch die neueste Forschung weitgehend bewahrt hat, wenn auch nicht immer unter Beachtung des 'polyphonen $<$ Charakters ihrer Beziehung.

Neben den engen Banden, die Basilius mit diesen beiden Theologen verknüpfte, haben wir aus seinem Briefwechsel mehr oder weniger zahlreiche, mehr oder weniger belastbare Informationen über sein Verhältnis zu einer großen Zahl weiterer kirchlicher Gestalten der Zeit. Da sind zunächst einmal Meletius, Patriarch von Antiochia und Eustathius, Bischof von Sebaste, beide zumindest zeitweise enge Gefährten des Basilius, beide aber auch für sich genommen bedeutende Theologen und Kirchenmänner, wenn auch für uns wegen des Quellenmangels nur schattenhaft zu erfassen. Aus theologiegeschichtlicher Sicht sicherlich noch interessanter sind die Schlaglichter, die die Basiliuskorrespondenz auf dessen Verhältnis zu Athanasius von Alexandria und dessen Freund, den später verurteilten Apolinarius von Laodicea wirft. Zumindest punktuell erfahren wir aus den Briefen auch etwas über die Verbindungen, die Basilius mit dem religiösen Eiferer Epiphanius von Salamis unterhielt, über seine Kontakte mit den konservativen, so genannten homöusianischen Bischöfen um Basilius von Ankyra sowie über seine Bekanntschaft mit dem origenistischen Theologen und Asketen Evagrius Ponticus.

All diese Informationen also erlauben es uns, Basilius' Einbindung in die komplexen theologischen und kirchlichen Netzwerke seiner Zeit besser zu erfassen und zu verstehen als uns das für die übergroße Mehrheit der Kirchenväter möglich ist. Gleichzeitig jedoch entzieht Basilius sich unserem Verständnis auch sehr entschieden,

1. Griechische-französische Ausgabe: Y. Courtonne, Saint Basile. Lettres, 3 Bd., Paris, 1957-66. Deutsche Übersetzung mit ausführlichem Anmerkungsapparat: Basilius von Caesarea. Briefe, übers. W.-D. Hauschild, 3 Bd., Stuttgart 1973-93.

2. R. Pouchet, Basile le Grand et son univers d'amis d'après sa correspondence, Rom 1992. Die bis heute maßgeblichen Untersuchungen zur Kirchengeschichte des vierten Jahrhunderts von Eduard Schwartz und Hans Lietzmann beruhen für die 370er Jahre ebenfalls weitgehend auf aus den Basiliusbriefen gewonnenen Informationen.

3. Der Tod des Basilius wurde traditionell auf den 1. Januar 379 datiert, aber die neuere Forschung hat gezeigt, dass er wohl einige Monate früher gestorben sein muss. Für einen Forschungsüberblick zu dieser Frage vgl. A. Silvas, Gregory of Nyssa. The Letters, Leiden 2007, 32-39. In jedem Fall erlebte der Bischof die große kirchengeschichtliche Wende nicht mehr, die im Jahr 379 der Regierungsantritt von Kaiser Theodosius I brachte und durch die sein Wirken für die Nachwelt so erfolgreich erscheint. 
und das gerade in seiner 'polyphonen Interaktion mit seinen Freunden, Kollegen und Rivalen. Wo genau seine theologischen und intellektuellen Wurzeln und Bindungen liegen, ist deshalb oft erstaunlich schwer festzumachen. Anscheinend verband ihn mit seinem Freund Gregor von Nazianz ein gemeinsames Interesse an dem großen Denker des dritten Jahrhunderts, Origenes von Alexandria, ${ }^{4}$ aber die theologischen Ideen des Origenes und sein wissenschaftlicher Ansatz sind bei Basilius weit weniger präsent als bei seinem Freund oder auch bei Gregor von Nyssa. Das ist besonders deutlich in seinen Homilien zum Sechstagewerk, einer Schrift, die von der gesamten griechischen Kirche als maßstabsetzend für eine christliche Aneignung der griechischen wissenschaftlichen Bildung angesehen wurde. Von der einflussreichen Genesisauslegung des Origenes jedoch zeigt sich bei Basilius kaum etwas, und die umstrittene allegorische Methode des Alexandriners, die Basilius` Bruder Gregor ausdrücklich verteidigte, wird von jenem explizit abgelehnt.

Gelegentlich hat man versucht, die Trinitätslehre des Basilius auf die vermeintlich origenistische Sichtweise der Homöusianer zurückzuführen, jedoch ist eine solche historische Herleitung auch eher fragwürdig. In Wahrheit ist, wie wir noch sehen werden, Basilius gerade in seinem trinitarischen Denken erstaunlich originell. Es ist bemerkenswert, wie wenig dieser zweifellos einflussreichste Aspekt seiner Theologie von den Prinzipien und Ideen bewahrt, die zuvor als weithin anerkannt galten. Während er dem Athanasius als einem Pfeiler der nicänischen Orthodoxie zweifellos Respekt zollte, entfernte er sich in seinem trinitarischen Ansatz vom Denken des Patriarchen von Alexandria ebenso wie von der Tradition einer in drei Hypostasen ontologisch abgestuft realisierten Trinität, an der viele seiner orientalischen Freunde festhielten.

Diese Beobachtungen bestätigen sich dem umfassenderen Blick auf das Leben und Werk des Basilius. Der Bischof von Cäsarea scheint sich regelmäßig in einer betonten Distanz von den konventionellen Entwicklungslinien und Kategorien zu befinden, durch die man die patristische Theologie gewöhnlich strukturiert. Er gehörte zu keiner Schule, und er begründete auch keine. Sein Appetit für spekulative Ideen und seine theoretische Neugier waren erstaunlich begrenzt; zumindest hatte er kein Bedürfnis von diesen Mitteilung zu machen. Im Gegenteil: er war extrem geschickt darin, sein Gegenüber nicht an allen seine Überzeugungen und Überlegungen teilhaben zu lassen. $\mathrm{Zu}$ vielen theologischen und philosophischen Fragen kennen wir seine Ansicht daher überhaupt nicht, was die Ortung seines Denkens innerhalb des intellektuellen Kosmos' der Welt der Patristik nicht eben einfacher macht. Fragt man beispielsweise nach seiner Christologie - nicht eben ein Nebenschauplatz der altkirchlichen Dogmenentwicklung! -, wird man bei Basilius kaum fündig, auch wenn viel dafür spricht, dass er auch in dieser Frage auf eher anderen Wegen wandelte als Athanasius vor ihm es getan hatte.

Diese Schwierigkeiten bei der Bestimmung des Parts, den Basilius in der Polyphonie der Väter gespielt hat, kann nur zum geringeren Teil der schwierigen Quellenlage angelastet werden. Immerhin wissen wir über Basilius weitaus besser Bescheid als über die überwiegende Mehrheit der frühkirchlichen Denker. Man gewinnt vielmehr den Eindruck, dass Basilius im Bewusstsein seiner aristokratischen Statur und seiner

4. Die Forschung hat lange gemeint, dass die erhaltene Blütenlese oder Philokalia aus Texten des Origenes die Frucht dieser Zusammenarbeit war, aber diese Ansicht ist wohl nicht aufrecht zu erhalten: N. McLynn, What was the »Philocalia of Origen «?, in: Meddelanden från Collegium Patristicum Lundense 19, 2004, 32-43. 
elitären Bildung ganz bewusst Distanz hielt von dem Lärm und der Hitze der zeitgenössischen Kontroversen, es sei denn seine Beteiligung war absolut erforderlich. Ein klassisches Beispiel für diese Tendenz findet sich in seinem 258. Brief, adressiert an Epiphanius von Salamis, den notorischen Ketzerjäger, dessen Persönlichkeit in jeglicher Hinsicht den stärksten denkbaren Kontrast zu der des Basilius bildet. Epiphanius hatte von christologischen Differenzen unter Mönchen auf dem Ölberg erfahren und war der Meinung, dagegen müsse etwas unternommen werden. Für diesen Plan versuchte er den Basilius zu gewinnen. Dieser freilich war alles andere als begeistert von dem Versuch, vor diesen Karren gespannt zu werden. Kühl informiert er den eifernden Epiphanius, er selbst sei unzureichend geschult, um solch komplexe dogmatische Probleme voll zu verstehen. Den Vorschlag zumal, man müsse dem nicänischen Bekenntnis eine christologische Formel hinzufügen, lehnt Basilius kategorisch ab:

Die jenem Glaubensbekenntnis (sc. dem Nicänum) hinzugefügten Lehren über die Menschwerdung des Herrn haben wir als unser Begreifen übersteigend weder traktiert noch akzeptiert, wie wir wissen, dass wir dann, wenn wir von der Einfachheit des Glaubens einmal abgehen, überhaupt kein Ende der Diskussion finden werden, da stets der Widerspruch uns weiter vorantreibt, und dass wir die Seelen der einfachen Gläubigen durch die Einführung fremder Dinge verwirren werden. ${ }^{5}$

Bedenkt man die destruktiven Energien, die die christologische Kontroverse in der östlichen Kirche seit dem fünften Jahrhundert entfesselt hat, kann man der Weigerung des Basilius, hier eine neue Front in der Glaubenskontroverse aufzumachen, die Bewunderung kaum versagen. Wie dem aber auch sei, mir geht es hier einzig um seine bemerkenswerte Fähigkeit, sich eines theologischen Urteils zu enthalten und seine ebenso bemerkenswerte Bereitschaft, dies im entscheidenden Moment auch zu tun. Von beidem legt der zitierte Passus eindrücklich Zeugnis ab. Aus dem gesamten Abschnitt lässt sich nicht im Entferntesten entnehmen, welche Position Basilius selbst zu dem dogmatischen Streit der Ölbergmönche einnahm, über den er im Übrigen nicht nur durch Epiphanius, sondern auch durch ihm befreundete Mönche aus Palästina direkt unterrichtet war.

Diese Tendenz des Basilius sich vor unkluger Entblößung in dogmatischen Fragen zu hüten, muss in Betracht gezogen werden, will man seine Stimme im Chor der antiken christlichen Autoren identifizieren. Die Zurückhaltung des Basilius und die bewusste Distanz, die er zu seinen Freunden und Partnern wahrt, machen ein solches Unternehmen schwierig. Dieses Problem lässt sich dann lösen, wenn man in dieser Tendenz eine bewusste Haltung wahrnimmt, die ihrerseits auf eine ganz eigentümliche Persönlichkeit verweist, die unter den zahlreichen aufgeregten Stimmen der Zeitgenossen durch eine überlegene Ruhe und intellektuelle und spirituelle Souveränität hervorstach. Durch eben diese Persönlichkeitsmerkmale hat, so meine ich, Basilius seine einmalige Wirkung ausgeübt. Auch wenn sein Einfluss auf die dogmatische Entwicklung bedeutend war, lässt sich doch die These vertreten, dass die einzigartige Achtung, die ihm schon zu seinen Lebzeiten und besonders nach seinem Tod entgegengebracht wurde, durch diese persönlichen Eigenschaften bedingt war. Mein Versuch, mich seiner Person zu nähern, wird daher im Folgenden an diesem Punkt ansetzen.

5. Basilius, Ep. 258, 2, 20-27 (hg. Y. Courtonne, Bd. 3, 101). Übersetzung: W.-D. Hauschild, Briefe, Bd. 3, 104. 


\section{Theologie im Dienst kirchliche Praxis}

Basilius war zweifellos ein Mensch mit enormen intellektuellen Gaben und Fähigkeiten. Soweit unsere Quellen uns dies Urteil erlauben, muss er auch als einer der gebildetsten Repräsentanten der christlichen Kirche bis zum Ende des vierten Jahrhunderts gelten. Dazu leistete zweifellos sein aristokratischer Hintergrund einen Beitrag. Die Familie, der Basilius entstammte, war eine der reichsten der Provinz Kappadokien in der heutigen Türkei; ihre Bekehrung zum Christentum reichte in die vorkonstantinische Zeit zurück. Unter seinen zugegebenermaßen zahlreichen Geschwistern wurden drei später als Heilige anerkannt: zusätzlich zu Basilius und Gregor von Nyssa auch deren ältere Schwester Makrina. Basilius ragte also von vornherein aus der Menge seiner christlichen Zeitgenossen heraus. Seine einmalige Bedeutung in der Ostkirche jedoch verdankt er dem Zusammenstimmen seiner großen intellektuellen Gaben, seines religiösen Hintergrundes und seiner sozialen Statur mit einer ganz außergewöhnlichen Fähigkeit als Organisator und Kommunikator. Diese spezifische Kombination von Gaben und Fähigkeiten ist in der Geschichte der christlichen Kirche extrem selten. Das Christentum hat tiefe, grübelnde und bohrende Denker in großer Zahl hervorgebracht. Ebenso wenig hat es in seiner Geschichte an prophetischen und visionären Gestalten gemangelt, die oft ihr ganzes Leben einer einzigen Idee hingaben. Auch Ireniker, die den Konflikt scheuten und ihre Energien auf die Vermittlung tiefsitzender Gegensätze und Feindschaften verwandten, sind in ihr keine Seltenheit. Basilius hingegen war anders: ein vollkommen nüchterner Pragmatiker mit beträchtlichem Charisma und natürlicher Autorität begabt, gleichzeitig aber auch ein Visionär. Basilius war kein Taktiker und kein machiavellischer Intrigant, auch wenn er sehr wohl bereit war, beim Hobeln gelegentlich die sprichwörtlichen Späne fallen zu lassen. Er zögerte nicht, seine Gegner zu stigmatisieren oder zu Sündenböcken zu stilisieren; gelegentlich tat er dies sogar ehemaligen Freunden oder Mentoren an, wie im Fall des Eustathius von Sebaste und des Apollinarius von Laodicea. Man bekommt jedoch nie den Eindruck, dass er aus Boshaftigkeit oder Hass handelt; die Brutalität und Feindseligkeit, die man bei einem Athanasius oder auch bei Martin Luther findet, waren dem Basilius fremd. Der Grund hierfür scheint darin zu liegen, dass seine Vision letztlich mehr praktisch als dogmatisch war. Konkret war es die Notwendigkeit kirchlicher Einigung nach den traumatischen Konflikten und Spaltungen durch die trinitarische Kontroverse seit den 320er Jahren, der Basilius sich verschrieb und die er unermüdlich vorantrieb.

In der vorangegangenen Generation hatte Athanasius am Bekenntnis von Nicäa stur und unnachgiebig festgehalten; zu dem Zeitpunkt, als Basilius die kirchenpolitische Bühne betrat, hatte diese Taktik jedoch zu einer praktisch kompletten Isolierung des alexandrinischen Patriarchen geführt. Ein Vergleich der beiden Persönlichkeiten ist faszinierend: Beide haben zweifellos viel gemein, nicht nur das Bekenntnis zum Glauben von Nicäa, sondern auch starkes persönliches Charisma; beide waren Asketen mit ausgeprägtem Organisations- und Kommunikationstalent. Und doch könnten zwei Menschen kaum unterschiedlicher sein: Während Athanasius spaltete, war Basilius ein Versöhner und Heiler. Athanasius polarisierte; er wurde gleichzeitig bewundert und gehasst. Im Gegensatz dazu scheint sich Basilius allgemeiner Liebe und Verehrung erfreut zu haben; es spricht für sich, dass ihm bereits zu Lebzeiten der Beiname »der Große« beigelegt wurde.

Damit ist keinesfalls gesagt, dass die übermächtige Persönlichkeit des Basilius immer leicht zu ertragen war. Sowohl Gregor von Nazianz als auch Gregor von Nyssa 
legen auf je unterschiedliche Art Zeugnis von der geradezu erstickenden Macht seiner Gegenwart ab. Dies jedoch tat offenbar ihrer Verehrung für die Person des Basilius keinen Abbruch; und das, so meine ich, zu recht. Die Persönlichkeit des Basilius hatte etwas von einer Naturgewalt, die unvermeidlich jeden, der mit ihr zu tun hat, in ihren machtvollen Bann zieht. Man kann daher verstehen, dass diejenigen, die sich regelmäßig in seiner Nähe aufhielten, seine Wirkung als überwältigend empfanden. Dies erst recht dann, wenn sie selbst ungewöhnlich begabte und sensible Menschen waren. Aber Basilius tat dies nicht mit Mutwillen oder aus unlauterer Absicht; es war eine Wirkung, die sich gewissermaßen zwangsläufig durch seine Persönlichkeitsstruktur ergab und die von seinen Freunden als solche offenbar erkannt und anerkannt wurde.

Die Beiträge des Basilius zur Trinitätstheologie und insbesondere zur Lehre vom Heiligen Geist waren von größter Bedeutung, aber sie waren Teil seiner praktischen Vision für die Einigung der Kirche seiner Zeit. Seine Gaben befähigten ihn diese Aufgabe zu erfüllen, und er bediente sich ihrer mit Geschicklichkeit und mit oft erstaunlichem Erfolg. Die Größe und Bedeutung seines letztlich errungenen Triumphes können kaum überschätzt werden, auch wenn die persönliche Tragik des Basilius darin liegt, dass er starb, bevor dieser Triumph sich auch nur andeutete. Sein unvergleichliche Ruf in der östlichen Kirche verdankte sich zum nicht geringen Teil dieser Erfolgsgeschichte: Das späte vierte Jahrhundert bot den seltenen Fall einer gespaltenen Kirche, die auf der Grundlage dogmatischer Übereinkunft wieder zur Einheit zurückfand. Dies geschah nicht ohne die gezielte Exklusion einiger wichtiger Gestalten der vorausgegangenen Jahrzehnte: Eunomius von Cyzikus, Apolinarius von Laodicea und Markell von Ankyra, um nur die wichtigsten zu nennen. Basilius war bereit, diesen Preis zu bezahlen; an zahlreichen Stellen illustrieren seine Briefe die problematische Rolle, die der Exklusion einzelner für Gemeinschaftsbildung und soziale Kohäsion gerade in der christlichen Kirche zugekommen ist. ${ }^{6}$ Und doch - auf die Gefahr hin zu apologetisch zu klingen, sei es gesagt - geht Basilius selbst mit diesen Fällen erstaunlich souverän und mit einer gewissen Größe um, als sei ihm bewusst, dass es sich hier um ein Opfer handelt, das für ein höheres Gut notwendig ist, das er selbst gleichwohl nur ungern darbringt. ${ }^{7}$

\section{Eine neue Trinitätstheologie}

Mit all dem ist nun keinesfalls gesagt, dass Basilius nur Organisator und pragmatischer Kirchenpolitiker war. Sein seltenes Genie bestand vielmehr darin, dass er diese Gaben mit einem hohen Grad intellektueller und theologischer Originalität zu verbinden wusste. Sein wichtigster Beitrag ist zweifellos die Neuorientierung der Trinitätstheologie, die er eingeleitet hat. Es ist im Grunde schwer vorstellbar, dass die Arbeit einer einzelnen Person in diesem theologischen Feld, das ja beim Eintritt des Basilius in die Diskussion bereits seit mindestens 150 Jahren im Zentrum patristischer Debatten in der Ostkirche gestanden hatte, zu einer derart radikalen Richtungsänderung der dogmatischen Entwicklung führen konnte. Und doch ist dies eine richtige, wenn auch natürlich vereinfachte Beschreibung dessen, was geschah.

6. J. Zachhuber, The Rhetoric of Evil and the Definition of Christian Identity, in: P. Fiddes/J. Schmidt (Hgg.), Rhetorik des Bösen - Rhetoric of Evil, Würzburg 2013, 193-217.

7. Vgl. hier R. Girards Interpretation von Joh. 11, 50: The Scapegoat, übers. Y. Freccero, Baltimore 1986, 112-114. 
Der von Basilius eingeführte trinitarische Ansatz wurde, wenngleich vertieft und modifiziert von den anderen Kappadoziern, zum allgemein anerkannten Paradigma östlicher Trinitätslehre seit dem Ende des vierten Jahrhunderts. Bereits im fünften Jahrhundert setzen die großen Kontrahenten der Zeit, Kyrill von Alexandria und Nestorius von Konstantinopel, das kappadozische Modell als selbstverständlich voraus. Seine terminologischen und philosophischen Grundlagen wurden darüber hinaus auch auf andere wichtige dogmatische Fragen angewandt, etwa die Schöpfungs- und die Erlösungslehre; für den christologischen Streit waren diese Prinzipien ebenfalls grundlegend, führten allerdings zu weitreichenden konzeptionellen Problemen. ${ }^{8}$ Dies liegt jedoch außerhalb meines Themas in diesem Beitrag.

Wie konnte es dazu kommen? Man kann natürlich sagen, dass Basilius mit seiner trinitarischen Lehre einfach die Wahrheit des christlichen Bekenntnisses auf den Begriff brachte. Sucht man jedoch nach einem anderen Typ von Antwort, dann wird man schnell wieder auf den Kairos geführt, den magischen Zeitpunkt, zu dem diese neue Theorie geprägt wurde, sowie ihre Rolle bei der Beendigung eines bitteren, die Kirche tief spaltendend Disputes: diese Faktoren müssen gemeinsam mit der unbestreitbaren konzeptionellen Stärke des basilianischen Ansatzes in Betracht gezogen werden, will man seinen durchschlagenden Erfolg verstehen.

Worin bestand nun dieser Ansatz und inwiefern war er neu? Grob ausgedrückt und für den Moment die spolyphone Dimension alles patristischen Denkens außer Acht lassend - lässt sich sagen, dass die große Mehrheit patristischer Autoren im dritten und vierten Jahrhundert die Gottheit Jesu Christi, des präexistenten Logos, als eine abgeleitete verstanden. Der eine Gott, von dem die Schrift sprach, war Gott der Vater. Sollte der Sohn ebenfalls Gott sein, ohne dass dadurch der Monotheismus außer Kraft gesetzt wurde, dann konnte seine Gottheit nur so erklärt werden, dass sie aus der Göttlichkeit des Vaters entsprungen war und auf diese Weise auch mit ihm verbunden blieb. Diese Sichtweise wurde einflussreich von Origenes von Alexandria artikuliert und entwickelt (mit einigen Anleihen in der platonischen Ontologie); dennoch sollte man mit dem Begriff Origenismus in diesem Zusammenhang vorsichtig sein. Denn dies derivative Modell wurde in jener Zeit nicht als Ausdruck einer Schulzugehörigkeit empfunden; vielmehr war es ein allgemein anerkanntes Axiom trinitarischen Denkens, das wir daher sowohl bei Anhängern des nicänischen Bekenntnisses wie Athanasius und Apolinarius als auch bei ihren Gegnern finden.

Der Name des Apolinarius ist in diesem Zusammenhang von besonderer Bedeutung, denn der Briefwechsel zwischen ihm und dem jungen Basilius von Cäsarea Anfang der 360er Jahre ist eines der ersten Anzeichen für den bevorstehenden Paradigmenwechsel. Diese Korrespondenz ist gelegentlich als gefälscht verdächtigt worden, aber es gibt gute Gründe für ihre Echtheit. Basilius ist zu jenem Zeitpunkt noch unsicher, ob er das Bekenntnis von Nicäa anerkennen soll und wie es zu verstehen sei. Insbesondere trifft das auf den Kern der Kontroverse zu, das Wort homoousios, wesensgleich. Basilius trägt eine Reihe von bekannten Einwänden gegen den Gebrauch dieses Begriffs vor und fährt dann wie folgt fort:

Wir aber sind der Ansicht, dass all das, was man für das Wesen (ousia) des Vaters hypothetisch annehmen könnte, ebenso für das Wesen des Sohnes angenommen werden muss. Wenn man

8. J. Zachhuber, Christology after Chalcedon and the Transformation of the Philosophical Tradition, in: M. Knežević (Hg.), The Ways of Byzantine Philosophy, Alhambra 2015, 89-110. 
also daher das Wesen des Vaters als »vernünftiges, ewiges, ungezeugtes Licht « bezeichnet, dann wird man das Wesen des Sohnes ebenfalls »vernünftiges, ewiges, gezeugtes Licht « nennen. In diesem Sinn jedoch scheint mir der Begriff »ähnlich ohne Unterscheidung « besser zu passen als das Wort homoousios. ${ }^{9}$

Basilius ist also, wie man sieht, zu diesem Zeitpunkt (kurz nach 360) erst auf dem Weg dahin, das Bekenntnis von Nicäa anzunehmen. Dies ist angesichts des theologischen Klimas der Zeit weniger bemerkenswert als es im Rückblick erscheinen könnte. Viel eher verdient unsere Aufmerksamkeit, dass sich in diesen wenigen Zeilen bereits der Nukleus der kappadozischen Trinitätslehre deutlich ankündigt. Die Neuartigkeit dieses Modells liegt darin, dass es sich deutlich vom bislang herrschenden derivativen Modell absetzte. Während dieses Modell, wie gesehen, auf der Idee der ontologischen Ableitung des Sohnes aus dem Vater beruhte, liegt dem kappadozischen Paradigma der Gedanke der univoken Prädikation göttlicher Eigenschaften zu Grunde.

In dieser Theorie definieren Idiomata - so wird der terminus technicus lauten sowohl das, was den trinitarischen Personen gemeinsam ist, als auch das, was sie unterscheidet. Dies Modell ist in dem Schreiben des Basilius an Apolinarius noch nicht voll ausgearbeitet und doch eindeutig erkennbar. Die Art und Weise, in der Basilius über die Trinität denken und sprechen möchte, schließt selbstverständlich die traditionelle Annahme nicht aus, dass der Vater den Sohn zeugt und der Sohn vom Vater gezeugt wird. Entscheidend jedoch ist, dass diese beiden Begriffe (ungezeugt und gezeugt) nicht mehr so zur Definition der einen Gottheit gebraucht werden, dass der ungezeugte Vater der paradigmatische und eigentliche Gott ist, an dessen Göttlichkeit der Sohn durch seine Zeugung lediglich partizipiert. Stattdessen kommt beiden die Göttlichkeit gemeinsam zu, und diese ontologische Einheit wird dadurch zum Ausdruck gebracht, dass alle Prädikate, die wahrheitsgemäß vom Wesen des Vaters ausgesagt werden, ebenso auch vom Wesen des Sohnes prädiziert werden müssen. In diesem Sinn schreibt Basilius, dass »all das, was man für das Wesen (ousia) des Vaters hypothetisch annehmen könnte, ebenso für das Wesen des Sohnes angenommen werden muss."

Es geschieht in der Dogmengeschichte nur selten, dass man einer wirklich innovativen Idee begegnet, und wo dies geschieht, werden solche Neuerungen gewöhnlich aufs Schärfste abgelehnt und bekämpft. Warum geschah dies im Fall des Basilius nicht? Ich bin nicht sicher, dass es auf diese Frage jemals eine vollkommen befriedigende Antwort geben wird; die beste Erklärung ist vielleicht die folgende. Weiter oben war davon die Rede, dass die derivative Theorie bis zur Zeit des Basilius generell akzeptiert war. Von dieser allgemeinen Regel gab es allerdings eine wichtige Ausnahme, Eunomius von Cyzicus. Dieser extreme Vertreter einer >arianischen $>$ Vorordnung des Vaters, den er nur »der Ungezeugte « nannte, machte aus seiner Verachtung gegenüber dem derivativen Modell kein Geheimnis. Ein Großteil seiner scharfen und brillant vorgetragenen Polemik richtete sich gegen die Idee, der Sohn sei ein zweites göttliches Prinzip, ontologisch aus dem Vater hervorgegangen und deshalb ihm wesensähnlich. Aus unseren Quellen ist klar, dass das Denken des Eunomius allgemein als äußerst gefährlich angesehen wurde; die wichtigsten Theologen der Zeit - neben Basilius waren das Theodor von Mopsuestia, Apolinarius von Laodicea und Gregor von Nyssa - verfassten detaillierte Widerlegungen seiner Hauptschriften. Es ist daher 
wahrscheinlich, dass ein generelles Gefühl der Bedrohung durch dieses Denken die Bereitschaft genährt hat, eine neuartige, orthodoxe Ansicht der Trinität zu akzeptieren, die der Kritik des Eunomius effektiv Paroli bieten konnte - eben das bot der Ansatz des Basilius.

Damit soll nun keinesfalls gesagt sein, dass die basilianische Trinitätstheologie über jede Kritik erhaben war. Das größte Problem, das sich aus seinen Grundsätzen für die kirchliche Lehre ergab, war ihre Interpretation als Tritheismus. Der Vorwurf, dass die kappadozische Lehre dazu führe, dass Vater, Sohn und Geist drei Götter sind, wurde Basilius und seinen Mitstreitern bereits zu ihren Lebzeiten gemacht; zu einer wirklichen dogmatischen Kontroverse wuchs das Problem allerdings erst im sechsten Jahrhundert heran. Ein Leser, der bereit ist, sich durch die extrem langatmig verfasste, nur syrisch erhaltene, dreibändige Polemik Gegen Damian des Antiochener Patriarchen Petrus von Callinicum durchzuarbeiten, wird erstaunt sein angesichts der gründlichen und detaillierten Exegese von maßgeblichen Texten des Basilius, die sich dort findet. An ihr lässt sich sowohl die Größe seiner Autorität als auch die Ambivalenz seines theologischen Erbes ermessen. ${ }^{10}$

\section{Basilius als geistliche und kulturelle Autorität}

Von Basilius als einem Visionär war bereits die Rede. Doch war die Wiederherstellung der kirchlichen Einheit nicht die einzige Vision, die der Kappadozier verfolgte. Ebenso bedeutend war sein klarer Sinn dafür, dass im neuen Zeitalter eines zunehmend christianisierten Reiches, grundlegende Fragen des christlichen Lebens und der geistlichen Existenz ebenso neuer Antworten bedurften. Es kann heute leicht geschehen - insbesondere im Kontext theologischer Forschung -, dass die Bedeutung des Basilius auf seine dogmengeschichtlichen Neuerungen sowie seine Rolle in der Kirchenpolitik der Zeit reduziert wird. Für seine Zeitgenossen jedoch und für die byzantinische Kultur der nachfolgenden Jahrhunderte waren sein Beitrag als Organisator des Mönchstums und als Repräsentant eines gebildeten christlichen Humanismus von mindestens ebenso großer Signifikanz. Von dieser Tatsache bekommen wir einen Eindruck, wenn Photius, Patriarch von Konstantinopel im 9. Jahrhundert, bei der Beschreibung des basilianischen Stils ins Schwärmen gerät und meint, der Leser dieser Texte brauche weder Isokrates noch Platon, um den rhetorisch perfekten Gebrauch des Griechischen zu lernen. ${ }^{11}$ Ganz ähnlich liest man es im 12. Jahrhundert bei Michael Psellus: Ganz offensichtlich spielt für beide christliche Autoren eine Rolle, dass es christliche Autoritäten gibt, die sich in rhetorischer Bildung und im Gebrauch der griechischen Hochsprache nicht vor den paganen Vorbildern der klassischen Zeit verstecken müssen.

Nun war aber für die rhetorische Tradition die Fähigkeit des sprachlichen Ausdrucks eng mit der moralischen Kompetenz und der ethischen Valenz der Person verbunden. Der Rhetor war, in der berühmten Wendung, die von Quintilian dem Cato zugeschrieben wird, vir bonus dicendi peritus: ein guter Mann, der sich geschickt auszudrücken weiß. In diesem Sinn weist dieser allzu kurze Hinweis auf Basilius als

10. Auch für die hitzige frühneuzeitliche Debatte um den Tritheismus spielt das kappadozische Erbe eine erhebliche Rolle.

11. Photius, Bibliotheca 141. 
rhetorisches Idealbild zurück auf meine Ausgangsthese, nach der der Platz des Basilius in der Polyphonie der Väter insbesondere durch seine einzigartig faszinierende Persönlichkeit erklärbar ist.

\title{
Johannes Chrysostomus
}

\author{
Daniel Buda
}

\begin{abstract}
This paper contains a short biography of St. John Chrysostom, as well as a presentation of his main theological points. Je ist he most important Theologian of the so-called "Antiochian Theological School «. His writings are better known for their ethical and pastoral aspects, than for their speculative-dogmatical ideas. This paper presents the following aspects of John Chrysostom's theology and activities: Christology, ecclesiology, the hole of the Bible, the role of ethics, his portrait as pastor, the role of philosophy, John Chrysostom as supposed author of the most celebrated liturgy in the Eastern Orthodox Church.
\end{abstract}

\section{Kurzbiographie}

Johannes Chrysostomus (J. Chr. $)^{1}$, bekannt auch als Johannes von Antiochia, wurde um 349 in Antiochia am Orontes in einer hoch angesehenen Familie geboren. Sein Vater Secundus, der ein hoher Offizier der oströmischen Armee war, starb kurz nach seiner Geburt. J. Chr. wurde von seiner Mutter Anthusa christlich erzogen. Er studierte sowohl die christliche Lehre in einer von Diodor (der später Bischof von Tarsus wird) geleiteten asketischen Schule als auch Rhetorik, Philosophie und Rechtswissenschaft. Der bekannte heidnische Rhetor Libanius war sein Professor. Nach dem damals üblichen Katechumenat wurde J. Chr. mit 23 Jahren von Bischof Meletius von Antiochia getauft und im Jahre 371 zum Lektor geweiht. Nach einem asketischen Leben in der Stadt, schloss er sich 372, nach dem Tod seiner Mutter, den syrischen Mönchen an, die in den Bergen um Antiochia lebten. Er verbrachte dort vier Jahre in absoluter Isolation und biblischem Studium. Nachdem er aus gesundheitlichen Gründen nach Antiochia zurückgekehrt war, wurde J. Chr. 381 zum Diakon und 386 zum Priester ordiniert. Zwölf Jahre wirkte er als Priester und Stadtprediger unter der Leitung des Seniorbischofs Flavian von Antiochia.

1. Siehe J. Quasten, Patrology B. III, The Golden Age of Greek Patristic Literature From the Council of Nicaea to the Council of Chalcedon, Notre Dame 1991, 424-434, mit angegebener Literatur.

Evang. Theol. 79. Jg., Heft 5, S. 369-375, ISSN 0014-3502

(C) 2019 by Gütersloher Verlagshaus, Gütersloh, in der Verlagsgruppe Random House GmbH, München 